\title{
Aplicación subcutánea de dióxido de carbono para atenuación de cicatrices
}

\section{Subcutaneous $\mathrm{CO} 2$ application on scars attenuation}

\author{
Martha Arellano Salazar \\ ARSACIS SAC, Av. Paseo de la República 5610 of 304. Miraflores.
}

\section{Resumen}

El dióxido de Carbono (CO2), predomina en la naturaleza en estado gaseoso. Es un gas incoloro e inodoro, y es pieza clave del ciclo biológico del carbono, su concentración en el aire es $0.03 \%$.

El $\mathrm{CO} 2$ es uno de los componentes básicos en la homeostasis del cuerpo humano y es un elemento resultante del metabolismo celular. El CO2 es 20 veces más soluble en el agua que en el aire y de acuerdo con los estudios científicos no da lugar a embolia gaseosa clínicamente significativa. En la circulación sanguínea el dióxido de carbono es transportado hacia los pulmones por 3 vías: como ion bicarbonato $65 \%$, Co2 unido a proteínas especialmente la hemoglobina $25 \%$ y CO2 disuelto en plasma $10 \%$. [1]

En países europeos como Francia y Alemania, desde la edad media se conocen las propiedades curativas del dióxido de carbono en problemas circulatorios y de piel y desde 1920 aproximadamente se viene aplicando CO2 subcutáneo para tratamientos médicos [2]

Estudios actuales demuestran el efecto del dióxido de carbono en la microcirculación sanguínea [3-6] así como su efecto en el incremento del colágeno luego la inyección intradérmica del gas [7]. Este proceso de cicatrización inducido por CO2 está mediado por óxido nítrico [8-10].

Presento dos casos clínicos de pacientes que acudieron a consulta por presentar heridas cortantes, en el rostro ambas en región superciliar con un tiempo de enfermedad de 10 días el primer paciente y 30 días el segundo. Ambos varones sanos sin contraindicaciones para el tratamiento y ambas heridas habían sido suturadas, evidenciándose cicatriz reciente ligeramente deprimida.

Se aplicó dióxido de carbono subcutáneo con la técnica llamada carboxiterapia, usando el equipo Carboxiderm 1C producido por Tonederm (Brasil) con agujas de 30G/13 mm. El CO2 utilizado fue proporcionado por Praxair, con un certificado de conformidad de la pureza de 99,99\% (certificado $n^{\circ}$ 12/000063). La aplicación fue subcutánea e intradérmica tanto en la zona de la herida como en el área periférica a la misma, a un flujo máximo de $50 \mathrm{cc} / \mathrm{min}$ y a un volumen máximo de aplicación de $300 \mathrm{cc}$. La frecuencia de aplicación fue 1 sesión cada 1 a 4 semanas según la evolución del paciente.

Los resultados evidenciaron mejoría en la piel, con atenuación de la cicatriz inicial, con mayor rapidez y mejor resultado final en el caso de la herida más reciente. Los resultados coinciden con los hallados por Nach et al [11], en cuanto a mejora de la apariencia de la piel y la disminución de la cicatriz.

Considerando que la aplicación subcutánea de $\mathrm{CO} 2$, conocida como carboxiterapia, es segura y eficaz es un método que debería tenerse en cuenta como tratamiento de cicatrices recientes y antiguas para estimular un adecuado proceso de regeneración de piel y por ende de cicatrización, con un resultado final de atenuación de cicatrices residuales.

Descriptores: CO2, carboxiterapia, cicatrices, regeneración de piel

\begin{abstract}
Carbon dioxide (CO2), is found in nature in a gaseous state. It is a colorless, odorless, and is a key part of the carbon cycle, its concentration in the air is $0.03 \%$.

$\mathrm{CO} 2$ is one of the basic components in the homeostasis of human body and is a result of cellular metabolism. $\mathrm{CO} 2$ is 20 times more soluble in water than in air and according to many scientific studies do not leading to no clinically significant embolism. In the blood circulation the carbon dioxide is transported to the lungs by three routes: $65 \%$ as bicarbonate ion, $25 \%$ as $\mathrm{Co} 2$ bound to proteins, especially the hemoglobin and plasma CO2 dissolved in $10 \%$. [1]
\end{abstract}


In European countries like France and Germany, from the Middle Ages, are known the healing properties of carbon dioxide on circulation problems and skin, and since 1920 has been applied subcutaneous CO2 for medical treatment [2]

Current studies demonstrate the effect of carbon dioxide in blood microcirculation [3-6] and its effect on collagen increased after intradermal injection of the gas [7]. This healing process induced by $\mathrm{CO} 2$ is mediated by nitric oxide [8-10].

I present two cases of patients who came to the consulting room due stab wounds in the face both in superciliary region with a time of disease 10 days the first patient and 30 days the second. Both healthy men without contraindications for treatment and both wounds were sutured, showing fresh scar slightly depressed.

Carbon dioxide was applied subcutaneously with a technique called Carboxytherapy, using Carboxiderm 1C equipment produced by Tonederm (Brazil) 30G/13 mm needles. The CO2 used was provided by Praxair, with a conformity certificate of $99.99 \%$ purity (certificate $n^{0} 12 / 000063$ ). The subcutaneous and intradermal application was therefore in the wound area as a peripheral area thereof, to a maximum flow of $50 \mathrm{cc} / \mathrm{min}$ and a maximum of application volume of $300 \mathrm{cc}$. The application rate was 1 session every 1-4 weeks depending on the patient's progress.

The results showed improvement in the skin, with attenuation of the initial scar, faster and better final result in the case of the most recent wound. The results agree with those found for Nach et al [11], in terms of improving skin appearance and decreased scarring.

Whereas subcutaneous application of $\mathrm{CO} 2$, known as carboxytherapy, is safe and effective is a method that should be considered as a treatment of recent and old scars to stimulate a proper process of skin regeneration and healing therefore, with a final result attenuation of residual scars.

\section{Keywords: CO2, Carboxytherapy, Carbon dioxide Therapy, scars, skin regeneration}

\section{Introducción}

El dióxido de Carbono (CO2), predomina en la naturaleza en estado gaseoso. Es un gas incoloro e inodoro, y es pieza clave del ciclo biológico del carbono, su concentración en el aire es $0.03 \%$.

El CO2 es uno de los componentes básicos en la homeostasis del cuerpo humano y es un elemento resultante del metabolismo celular. El CO2 es 20 veces más soluble en el agua que en el aire y de acuerdo con los estudios científicos no da lugar a embolia gaseosa clínicamente significativa. En la circulación sanguínea el dióxido de carbono es transportado hacia los pulmones por 3 vías: como ion bicarbonato $65 \%$, el $25 \%$ del Co2 unido a proteínas, especialmente la hemoglobina y CO2 disuelto en plasma 10\%. [1]. En el cuerpo humano el dióxido de carbono cumple funciones clave tales como ser un componente fundamental en el intercambio gaseoso a nivel tisular y alveolar, estimula la zona respiratoria quimiosensible del bulbo raquídeo pues es capaz de atravesar fácilmente la barrera hematoencefálica, además es uno de los compuestos químicos con efecto vasodilatador moderado en la mayoría de tejidos. El dióxido de carbono se viene utilizando ya desde varios años en el campo médico como agente insuflador en Cirugía laparoscópica, agente de contraste en Radiología intervencionista y agente de insuflación en cirugías cardíacas a cielo abierto para prevención de infecciones. En países europeos como Francia y Alemania, desde la edad media se conocen las propiedades curativas del dióxido de carbono en problemas circulatorios y de piel y desde 1920 aproximadamente se viene aplicando $\mathrm{CO} 2$ subcutáneo para tratamientos médicos por ejemplo como terapia coadyuvante para heridas crónicas y agudas de múltiples etiologías [2].

La aplicación subcutánea de dióxido de carbono se conoce como Carboxiterapia y debe su nombre a Luigi Parassoni quien en 1995 acuñó el nombre durante el XVI Encuentro Nacional de Medicina Estética, realizado en Roma por la sociedad italiana de Medicina Estética. En Italia desde 1993 bajo la dirección de la escuela italiana de la Universidad de Siena, varios científicos han aportado estudios clínicos de gran importancia sobre la eficacia de Carboxiterapia en tratamientos médicos diversos. Esta técnica está siendo utilizada e investigada actualmente en países europeos, asiáticos y en Latinoamérica, Brasil es el pionero. Perú está iniciando el camino. Su aplicación y su recuperación como herramienta para el área médica son de gran importancia, para tal caso presento dos casos clínicos sobre el efecto de carboxiterapia en la regeneración d piel en cicatrices. 


\section{Casos clínicos, material y métodos utilizados}

\section{Caso 1}

Paciente varón de 41 años de edad, aparentemente sano; quien acude por presentar cicatriz en región superciliar izquierda, originada por impacto cabeza-cabeza durante partido de fútbol. La cicatriz tiene un tiempo de 10 días, había sido suturada con hilo nylon y los puntos habían sido retirados a los 7 días, tres días antes de consulta. Se planteó al paciente la posibilidad del uso del dióxido de carbono para regenerar la piel de la cicatriz en frente. Se realizó una historia clínica previa a fin de conocer el estado de salud del paciente, conocer enfermedades previas y establecer posibles contraindicaciones. Se expusieron los detalles del tratamiento y los posibles efectos adversos que pudieran presentarse. El paciente aceptó iniciar con el procedimiento.

Para el procedimiento se usó dióxido de carbono especial, apto para uso médico proporcionado por Praxair, con un certificado de conformidad de la pureza de 99,99\% (certificado $n^{\circ} 12 / 000063$ ). El equipo de carboxiterapia fue Carboxiderm 1C producido por Tonederm (Brasil). La zona de la herida fue previamente limpiada con gasa embebida con alcohol etílico medicinal de $70^{\circ}$. Se realizaron aplicaciones subcutáneas e intradérmicas tanto en la zona de la herida como en el área periférica a la misma y se usó para esto agujas finas de $30 \mathrm{G} \times 13 \mathrm{~mm}$. El flujo de gas máximo utilizado fue de $50 \mathrm{cc} / \mathrm{min}$, y el volumen máximo de aplicación fue de 300 cc por sesión. La frecuencia de las aplicaciones fue de una sesión cada 2 semanas las seis primeras sesiones y luego una sesión cada 4 semanas las últimas cuatro sesiones. El número total de aplicaciones fue de 10 sesiones.

\section{Caso 2}

Paciente varón de 28 años, aparentemente sano quien acude a la consulta médica presentando cicatriz en ángulo interno de ceja izquierda originado por asalto y golpe con arma blanca. La herida cicatrizada tiene un tiempo de 4 semanas. Según refiere el paciente la herida fue profunda y requirió de 4 puntos internos y 6 puntos superficiales, los mismos que habían sido retirados a los 7 días. Se realizó la evaluación y la historia clínica previa para establecer estado de salud y posibles contraindicaciones del paciente. Se expusieron los detalles del tratamiento así como efectos adversos. El paciente aceptó iniciar el tratamiento.
Para el procedimiento se usó dióxido de carbono especial, apto para uso médico proporcionado por Praxair, con un certificado de conformidad de la pureza de 99,99\% (certificado $n^{\circ}$ 12/000063). El equipo de Carboxiterapia fue Carboxiderm 1C producido por Tonederm (Brasil).

La zona de la herida fue previamente limpiada con gasa embebida con alcohol etílico medicinal de $70^{\circ}$.

Se realizaron aplicaciones subcutáneas e intradérmicas tanto en la zona de la herida como en el área periférica a la misma y se usó para esto agujas finas de $30 \mathrm{G} \times 13 \mathrm{~mm}$. El flujo de gas máximo utilizado fue de $50 \mathrm{cc} / \mathrm{min}$, y el volumen máximo de aplicación fue de 300 cc por sesión. La frecuencia de las aplicaciones fue de una sesión cada 2 semanas, y el número total de sesiones fue de tres.

\section{Resultados y discusión}

Los resultados del caso 1 se muestran en las Figs. 1 a 3 . Los resultados del caso 2 se muestran en las Figs. 4 a 5.

Nuestros resultados según los registros fotográficos mostrados, evidencian regeneración de la piel con disminución de la cicatriz, en el primer caso con una aproximación del $90 \%$. El efecto del dióxido de carbono se basa en su poder vasodilatador arterial, incluso en estados de arteriopatía previa (2).

El propósito de Carboxiterapia es mejorar o restaurar la función de microcirculación cuando está dañada, además los resultados de la administración de $\mathrm{CO} 2$ no se deben solamente a la mejora de los parámetros locales de circulación y la perfusión tisular, sino también al incremento de la presión parcial de oxígeno tisular (3). El efecto vasodilatador y de incremento del flujo sanguíneo tisular ha quedado demostrado en diversos estudios experimentales (4-6).

A nivel de piel se ha demostrado el incremento del colágeno tanto con la aplicación subcutánea como intradérmica, la primera genera aumento de la densidad del colágeno mientras que con la segunda se consigue según los estudios un colágeno más compacto, el uso de ambas aplicaciones valdría para conseguir regeneración de la piel en caso de heridas o cicatrices (7). 


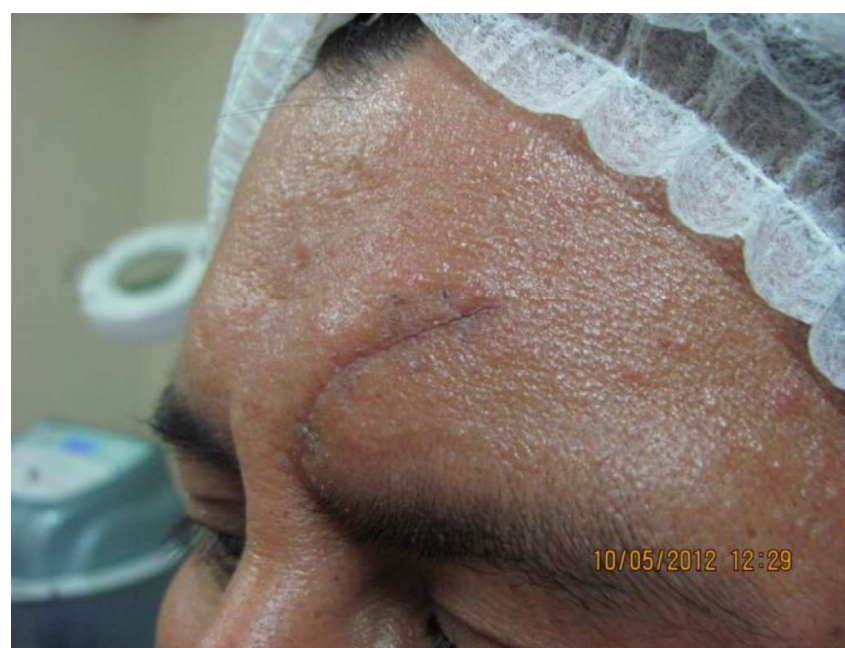

Fig. 1: Paciente caso 1 antes de inicio de carboxiterapia.

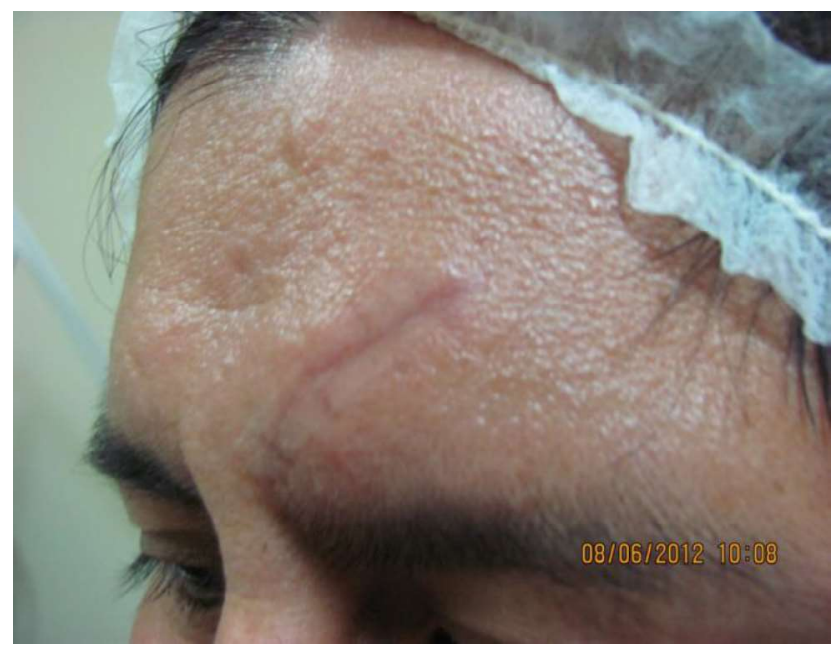

Fig. 2: Paciente caso 1, con tres sesiones de carboxiterapia.



Fig. 3: Paciente caso 1, con 10 sesiones de carboxiterapia.

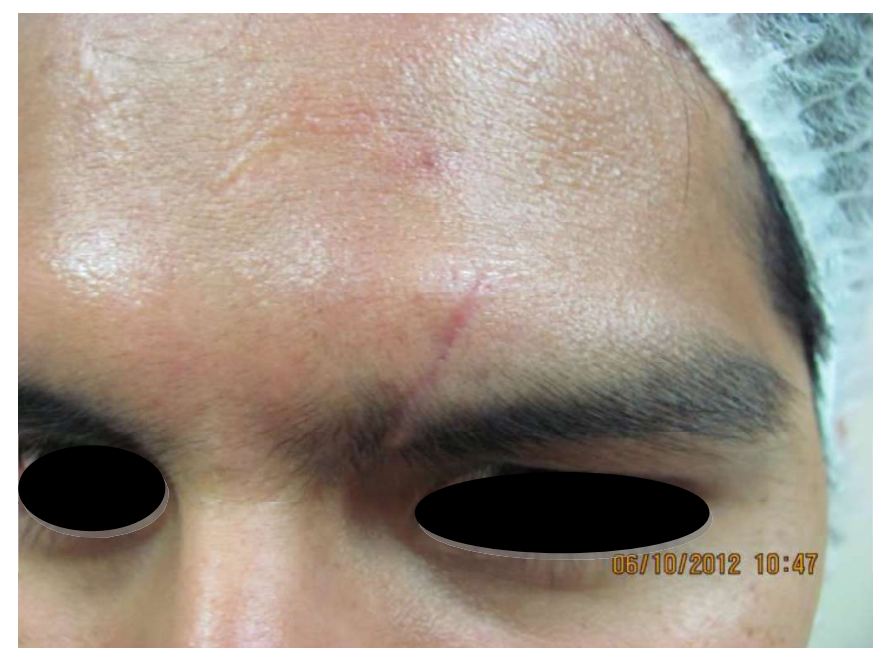

Fig. 4: Paciente caso 2, antes de inicio de tratamiento con carboxiterapia

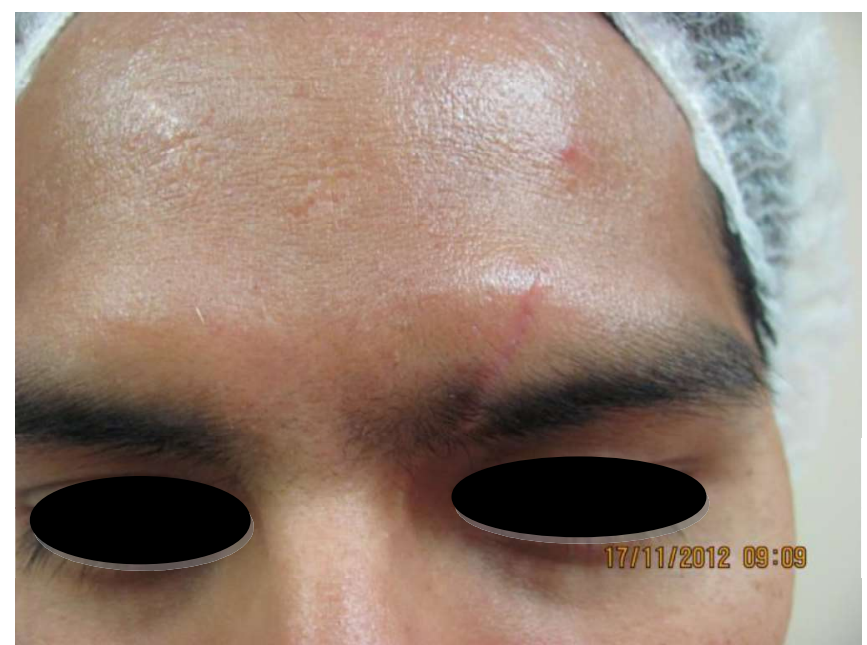

Fig. 5: Paciente caso 2, luego de 3 sesiones de tratamiento con carboxiterapia.

El efecto regenerador en piel, se explica a nivel de su efecto bioquímico en los tejidos (fig. 6), el año 2005 Irie et all demostraron por primera vez que el CO2 favorecería la inducción local del factor de crecimiento del endotelio vascular o VEGF (por sus siglas en inglés), esto a la vez se asocia con la producción de óxido nítrico en endotelio el mismo que está relacionado con el efecto vasodilatador observado además de la movilización de células progenitoras endoteliales y formación de nuevos vasos (8), otros efectos benéficos del óxido nítrico en la reparación de heridas se han atribuido a su influencia en angiogénesis, inflamación, proliferación celular, deposición de la matriz y remodelación $(9,10)$. Los resultados de ambos pacientes evidenciaron mejoría en la piel, con atenuación de la cicatriz inicial, con mayor rapidez y mejor resultado final en el caso de la herida más reciente. Los resultados coinciden con los hallados 
por Nach et al [11], en cuanto a mejora de la apariencia de la piel y la disminución de la cicatriz.

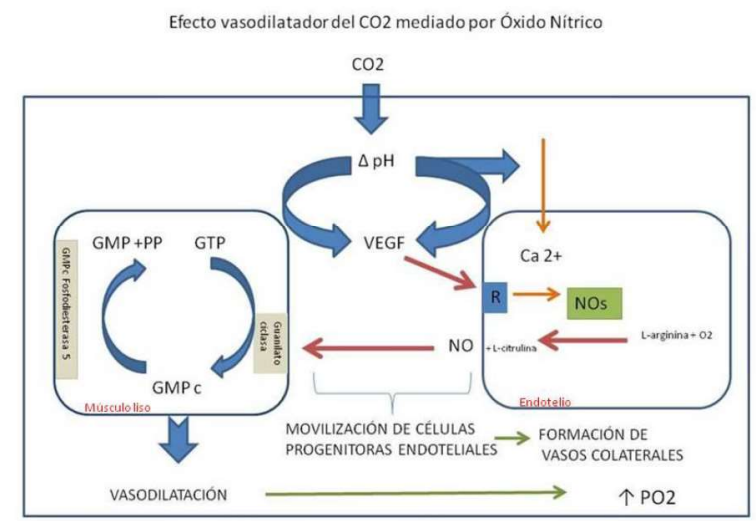

Fig. 6: Efecto vasodilatador del $\mathrm{CO} 2$ mediado por óxido nítrico. $\mathrm{CO} 2$ : dióxido de carbono, $\Delta \mathrm{pH}$ : variación de $\mathrm{pH}$, VEFG: factor de crecimiento del endotelio vascular, NO: óxido nítrico, NOs: óxido nítrico sintetasa, PO2: presión tisular de oxígeno.

\section{Conclusiones}

Carboxiterapia ha demostrado ser efectiva en atenuar cicatrices con mejor resultado en las más recientes. A mayor cantidad de sesiones, mayor atenuación de la cicatriz. Considerando que la aplicación subcutánea de $\mathrm{CO} 2$, conocida como carboxiterapia, es segura y eficaz es un método que debería tenerse en cuenta como tratamiento de cicatrices recientes y antiguas para estimular un adecuado proceso de regeneración de piel y por ende de cicatrización, con un resultado final de atenuación de cicatrices residuales.

\section{Agradecimientos}

A los pacientes que accedieron a la publicación de sus casos, pues contribuyen con la difusión de esta técnica.

\section{Referencias}

[1] Guyton A. y Heall J. Tratado de Fisiología Médica, Elsevier Science, 2006.

[2] C. Ambrosi, G. Delanoe, Ann. de Caridiologie et d'Angéiologie 1 (1976) 9398.

[3] V.Varlaro, et all., Acta Phleboligica 8 (2007) 3-11.

[4] T. Ito, J.I. Moore, M. C. Koss, The Journal of investigative Dermatology 93 (1989) 259262.

[5] W. Schnizer, R. Erdl, P. Schops, N. Seichert, Int. J Microcirc: Clin Exp 4 (1985)343-350.

[6] M. Minamiyama, A. Yamamoto, J Biorheol 24 (2010) 42-46.

[7] J. C. Tavares, A. Haddad, S Navarro, Journal of Drugs in Dermatology 7 (2008) 201-206.

[8] H.Irie et all., Circulation 111 (2005) 15231529.

[9] J. Luo, A. Chen Acta Pharmacologica Sinica 26 (2005) 259-264.

[10] S. Frank, H. Kämpfer, C. Wetzler, J. Pfeilschifter, Kidney International 61 (2002) 882- 888

[11] R. Nach, H. Zandifar, R. Gupta, J. S. Hamilton, Ear Nose Throat J 89 (2010) 6466

E-mail: paola@deperu.com 